\title{
ADVERSIDADE FAMILIAR E PROBLEMAS COMPORTAMENTAIS ENTRE ADOLESCENTES INFRATORES E NÃO-INFRATORES
}

\author{
Maria Delfina Farias Dias Tavares da Silva* \\ Maria Aznar Farias" \\ Edwiges Ferreira de Mattos Silvares" \\ Mariana Castro Arantes ${ }^{\infty}$
}

\begin{abstract}
RESUMO. O presente estudo comparou adolescentes infratores e não-infratores com relação a dois aspectos: grau de adversidade familiar e grau de concordância entre a autopercepção e a percepção dos pais sobre problemas de comportamento dos jovens. Participaram deste estudo 40 adolescentes, 23 atendidos no Centro de Atenção e Apoio ao Adolescente (UNIFESP) e 17 adolescentes considerados infratores sob intervenção da Vara da Infância e da Juventude da comarca de Santos. Os instrumentos utilizados foram o Child Behavior Checklist (CBCL), Youth Self Report (YSR) e o Índice de Adversidade Familiar de Rutter. O Índice de Adversidade Familiar do grupo de adolescentes infratores foi significativamente maior. Os resultados apontaram ainda que, embora a diferença não tenha sido estatisticamente significante, a porcentagem dos escores de discordância nas percepções sobre problemas de comportamento foi maior entre os adolescentes infratores e seus pais.
\end{abstract}

Palavras-chave: família, adolescentes, problemas comportamentais.

\section{FAMILY ADVERSITY AND BEHAVIOR PROBLEMS AMONG ADOLESCENT OFFENDERS AND NON-OFFENDERS}

\begin{abstract}
Young offenders and non-offenders are compared on two aspects: Family Adversity Index and Agreement Index between parents' perception and adolescents' self-perception on the behavior problem. Participants comprised 40 adolescents, or rather, 23 were being attended to at Attendance and Support Center for Young People (UNIFESP) and 17 adolescent offenders at the Court for the Welfare of Children and Young People in Santos SP Brazil. Child Behavior Checklist (CBCL), Youth Self Report (YSR) and Rutter's Family Adversity Index were employed. The family adversity index was significantly higher for young offenders. Results show that percentage of disagreement scores between the perceptions of behavior problems was higher, albeit not significant, between adolescent offenders and their parents.
\end{abstract}

Key words: Family, adolescents, behavior problems.

\section{ADVERSIDAD FAMILIAR Y PROBLEMAS DE COMPORTAMIENTO ENTRE ADOLESCENTES INFRACTORES Y NO-INFRACTORES}

RESUMEN. El presente estudio ha comparado adolescentes infractores y no-infratores con relação a dos aspectos: 1) Grado de adversidad familiar y 2) Grado de concordancia entre la autopercepción y la percepción de los padres sobre problemas de comportamiento de los jóvenes. Participaron de este estudio 40 adolescentes, 23 atendidos en el Centro de Atención y Apoyo al Adolescente (UNIFESP) y 17 adolescentes considerados infractores bajo intervención de la jurisdicción de la Infancia y de la Juventud de la comarca de Santos. Los instrumentos utilizados fueron el Child Behavior Checklist (CBCL), Youth Self Report (YSR) y el índice de adversidad familiar de Rutter. El índice de adversidad familiar del grupo de adolescentes infractores fue significativamente mayor. Los resultados apuntaron, aunque la diferencia no haya sido estadisticamente significante, que el porcentaje de las puntuaciones de discordancia en las percepciones sobre problemas de comportamiento fue mayor entre los adolescentes considerados infractores y sus padres.

Palabras-clave: Família, Adolescentes, Problemas de comportamiento.

\footnotetext{
* Mestre em Ciências Aplicadas a Pediatria. Instituto de Estudos Sociais, Núcleo de Estudos Em Educação e Pró Sociabilidade, Santos-SP.

\# Doutora em Psicologia Clínica. Professora do Curso de Psicologia da Universidade Católica de Santos.

II Doutora. Professora da Universidade de São Paulo, São Paulo.

æ Psicóloga.
} 
A associação entre determinadas características familiares e distúrbios psicológicos infantis é uma evidência empírica de aceite consensual. Essa associação é tão fortemente estabelecida que levou Rutter (1981) a circunscrever cinco fatores considerados adversos ao desenvolvimento infantil, os quais, em interação, podem concorrer para a manifestação de comportamentos disruptivos e delinqüentes. $\mathrm{O}$ conjunto desses fatores foi denominado pelo autor de Índice de Adversidade Familiar, e se inclui fatores: a) número excessivo de filhos na família, b) salário muito baixo dos pais; c) discórdia conjugal; d) número excessivo de pessoas residentes na mesma casa; e e) psicopatologia familiar. Independentemente do local onde resida a família (se em grandes centros urbanos ou em zonas rurais), os riscos de incidência de distúrbios infantis são mais prováveis quanto maior é o índice composto pela conjunção desses fatores.

Também no conhecido modelo desenvolvimentista do comportamento anti-social de Patterson, Reid e Dishion (1992), a família tem papel fundamental no aparecimento de problemas de conduta. O modelo foi descrito a partir de várias pesquisas empíricas e propõe que as práticas parentais pobres, em termos de disciplina e monitoramento durante os primeiros anos de vida, são condicionantes iniciais para o surgimento deste tipo de comportamento.

De acordo com o modelo desenvolvimentista do comportamento anti-social, quatro tipos de variáveis podem estar associadas a práticas parentais inadequadas: 1) características de comportamento dos avós (ex: comportamento anti-social e manejo familiar empobrecido); 2) características de comportamento dos pais (ex: comportamento anti-social e predisposto a estressores); 3) variáveis sociodemográficas (ex: baixa escolaridade, pertencer a grupo étnico minoritário); e 4) estressores familiares situacionais (desemprego, conflito marital e separação conjugal). Essas quatro variáveis, em interação, podem facilitar o isolamento social familiar, o que por si só já é um determinante de práticas educativas severas (Azevedo \& Guerra, 1989). Famílias com essas dificuldades são menos funcionais, menos positivas e menos consistentes, podendo facilitar o comportamento anti-social de seus membros, especialmente dos adolescentes (Silvares, 2000).

Segundo esse modelo, os problemas infantis decorrentes de uma família disfuncional podem levar a que a criança durante os anos da infância média seja rejeitada pelo grupo de pares não-desviantes e apresente dificuldades acadêmicas progressivas. Os problemas de conduta e rejeição pelos pares, inter- relacionados, aumentam a probabilidade de que a criança procure pares desviantes, ou seja, outras crianças que também tenham problemas de conduta. Uma das consequiências da associação com pares desviantes pode ser o encaminhamento para a delinquiência durante a adolescência.

O comportamento delinqüente constitui-se no agravamento de um padrão anti-social que se iniciou na infância e incluiria comportamentos desviantes e atos ilegais, como roubo, assalto, vandalismo e uso de drogas (Alvarenga et al., 2005).

Quando falamos em adolescente delinqüiente estamos nos referindo a uma combinação de vários comportamentos anti-sociais, passíveis de intervenção, mas nem sempre sob intervenção da justiça. $O$ adolescente delinqüiente passa a ser considerado um adolescente infrator no momento em que há a intervenção da lei, ou seja, no momento em que a justiça toma conhecimento de seus atos (Volpi, 1997).

Por vezes, $o$ ato infracional acontece isoladamente, mas em geral procede de uma história construída ao longo da vida do adolescente. Neste contexto, as condições emocionais, a capacidade de resiliência e as características do entorno próximo fatores peculiares a cada indivíduo - definem a maneira particular de enfrentar situações-problema (Silva \& Rossetti-Ferreira, 2002). A grande maioria dos adolescentes que apresentam comportamentos anti-sociais faz parte, sem dúvida, do universo de adolescentes destituídos de rede de apoio, de família formadora e continente e de recursos sociais e emocionais.

$\mathrm{Na}$ avaliação de problemas de comportamento de crianças e adolescentes em geral, tem-se considerado que o uso de múltiplos informantes, inclusive do autorelato, é capaz de fornecer um quadro mais detalhado do comportamento em diferentes contextos (Achenbach, 1991a). As discrepâncias nos relatos de comportamentos feitos por diversas fontes são inevitáveis e são indicadas por diversas pesquisas. Não há clareza, entretanto, quanto ao porquê dessas discrepâncias, nem quanto à existência de padrões de discrepância (Glaser, Calhoun, Bradshaw, Bates \& Socherman, 2000). As evidências sugerem que amostras clínicas e não-clínicas apresentam padrões distintos de concordância entre os informantes (Butler, Mackay \& Dickens, 1995).

Em um levantamento bibliográfico foram encontrados apenas dois estudos que investigaram discrepâncias no auto-relato de problemas de comportamento e no relato dos pais em amostras de adolescentes infratores (Butler et al., 1995; Glaser et al., 2000). Nos dois estudos, os pais reportaram 
significativamente mais problemas de comportamento do que os adolescentes infratores.

Apesar da discrepância entre o relato dos pais e o dos adolescentes infratores encontrada em um dos estudos, a freqüência de correlações acima da média entre os relatos dos pais e dos adolescentes foi mais elevada do que se poderia esperar em comparação com a amostra normativa (Glaser et al., 2000). No outro estudo, entretanto, a porcentagem de concordância no relato de problemas de comportamento entre as mães e os adolescentes infratores foi significativamente menor do que a porcentagem de concordância da amostra-controle (Butler et al., 1995).

Considerando a importância de ampliar a compreensão da população de adolescentes que se encontram sob intervenção da justiça, o presente estudo procurou comparar uma amostra de adolescentes infratores com uma amostra de adolescentes não-infratores com relação a dois aspectos: 1) Grau de adversidade familiar e 2) Grau de concordância entre a autopercepção e a percepção dos pais sobre problemas de comportamento dos jovens.

\section{MÉTODO}

\section{Participantes}

Participaram deste estudo 40 adolescentes e seus pais. Destes, 17 haviam cometido ato infracional e estavam sob intervenção da Justiça da Infância e Juventude da cidade de Santos.

Os outros 23 adolescentes participantes estavam sendo atendidos pela primeira vez em consulta médica, no Centro de Atenção e Apoio ao Adolescente (CAAA-UNIFESP), localizado em São Paulo. O CAAA é um centro de especialidades vinculado ao Departamento de Pediatria da Escola Paulista de Medicina - UNIFESP ${ }^{1}$.

Os critérios de inclusão na amostra de adolescentes infratores foram: 1)ter entre doze e dezoito anos de idade; 2) estar envolvido numa ação de ato infracional, de acordo com o artigo 103 da Lei 8.069/1990² (Estatuto da Criança e do Adolescente); e 3) estar acompanhado de um responsável legal. Para a amostra de adolescentes não-infratores foram: 1) ter entre onze e dezoito anos de idade (faixa etária atendida no CAAA; 2) não estar

Ainda que fosse desejável ter um grupo de comparação a mais, com adolescentes encaminhados para clínica-escola sem o encaminhamento médico de modo a eliminar o viés clinico, isso não foi possível por questões de ordem prática na época da coleta de dados.

2 Art. 103. Considera-se ato infracional a conduta descrita como crime ou contravenção penal. envolvido em prática de ato infracional - dado coletado em entrevista com os pais ou responsáveis; 3) estar acompanhado de um responsável legal; 4) estar na primeira consulta no CAAA.

\section{Instrumentos}

\section{Índice de adversidade familiar}

Para a avaliação do Índice de adversidade familiar, foi realizada uma entrevista estruturada que analisou cinco itens considerados adversos para o desenvolvimento infantil: número excessivo de pessoas na mesma casa; presença de discórdia conjugal; número excessivo de filhos; baixa renda mensal e existência de psicopatologia na família. Foram considerados como psicopatologia os casos de alcoolismo, adição às drogas, esquizofrenia e demais patologias mentais. Quanto maior o número de variáveis presentes, maior o grau de adversidade familiar. Para cada item de adversidade foram atribuídos 0,2 pontos, totalizando um escore máximo de um ponto e um mínimo de nenhum ponto. Foi considerado como de número excessivo de filhos o caso em que o número de filhos na família era maior que um desvio-padrão da média fornecida pela Fundação SEADE (1999) para a cidade em que a família reside (Santos ou São Paulo). O mesmo critério foi utilizado para o número excessivo de pessoas na família e para a baixa renda mensal.

\section{Child Behavior Checklist - Inventário de Comportamentos da Infância e da Adolescência (CBCL)}

O Inventário de Comportamentos da Infância e da Adolescência (CBCL) foi desenvolvido por Achenbach (1991a). O CBCL não tem normas brasileiras, mas existe um estudo preliminar de validação (Bordin, Mari \& Caieiro, 1995). Esse instrumento avalia a competência social e a presença de problemas de comportamento em crianças e adolescentes de acordo com a percepção dos pais.

A parte referente a problemas de comportamento compõe-se de 118 itens. Esses itens são agrupados em oito escalas-síndromes: I. Retraimento; II. Queixas somáticas; III. Ansiedade/depressão; IV. Problemas sociais; V. Problemas de pensamento; VI. Problemas de atenção; VII. Comportamento delinqüente; VIII. Comportamento agressivo; e IX Problemas sexuais. Os escores nas escalas I, II, e III agrupados compõem o escore de Distúrbios internalizantes (DI); os escores das escalas VII e VIII formam o escore de Distúrbios externalizantes (DE). A soma dos escores das oito escalas corresponde ao escore de Distúrbios totais (DT). 
Os escores podem ser considerados não-clínicos, clínicos e limítrofes. De acordo com o manual do Inventário (Achenbach, 1991a), os casos considerados limítrofes podem ser incluídos na categoria clínica para fins de pesquisa. Para os escores de soma de escalas (DI, DE e DT), os pontos de corte, de acordo com a orientação do manual do CBCL (Achenbach, 1991), determinam que o escore deve ser inferior a 60 para a categoria não-clínica, para a categoria limítrofe deve estar entre 60 e 63 e deve ser maior que 63 para categoria clínica, de acordo com normas americanas corroboradas pelo estudo de validação preliminar de Bordin et al. (1995).

\section{Young Self-Report (YSR)}

O Young Self Report (YSR) é um inventário de comportamentos auto-aplicável para jovens entre onze e dezoito anos. Trata-se de uma variação do CBCL também desenvolvida por Achenbach (1991b). A versão utilizada em nosso estudo foi a portuguesa, sem que houvesse a oportunidade de se promover a sua validação semântica

A somatória dos escores obtidos na segunda parte permite ao avaliador traçar um perfil comportamental do adolescente, derivado da análise de oito agrupamentos de itens ou síndromes: I. Retraimento; II. Queixas somáticas; III. Ansiedade/depressão; IV. Problemas com o relacionamento social; V. Problemas de pensamento; VI. Problemas de atenção; VII. Comportamento delinqüiente; VIII. Comportamento agressivo. Assim como no CBCL, os escores nas escalas I, II, e III agrupados compõem o escore de Distúrbios internalizantes (DI) e os escores das escalas VII e VIII formam o escore de Distúrbios externalizantes (DE). A soma dos escores das oito escalas corresponde ao escore de Distúrbios totais (DT). Os pontos de corte também são os mesmos. O resultado do YSR é ajustado ao sexo e à idade do adolescente.

\section{Procedimentos}

A amostra de adolescentes infratores foi aleatória, uma vez que os processos judiciais foram distribuídos para os vários membros da equipe técnica do Fórum da Comarca de Santos. Os adolescentes tiveram suas entrevistas agendadas com trinta dias de antecedência. Todos estavam envolvidos em delitos graves e eram reincidentes. A avaliação psicológica foi determinada pelo juiz da Vara da Infância e Juventude. Os adolescentes e seus pais passaram por entrevista e responderam ao CBCL e YSR.

Os adolescentes e pais atendidos no CAAA responderam ao YSR e ao CBCL por ocasião da primeira consulta no setor, independentemente da queixa ou da especialidade procurada.

\section{RESULTADOS}

\section{Dados sociodemográficos}

Tabela 1. Dados Sociodemográficos e p-valor do Teste T de Student ou do Teste de Fisher Para os Grupos de Adolescentes infratores e Não-Infratores.

\begin{tabular}{|c|c|c|c|}
\hline Variáveis & $\begin{array}{c}\text { Infrator } \\
\mathrm{N}=17\end{array}$ & $\begin{array}{c}\text { Não-infrator } \\
\quad \mathbf{N}=\mathbf{2 3}\end{array}$ & $\mathbf{p}$ \\
\hline Idade $(\operatorname{anos})^{* * * *}$ & 15,7 & 13,1 & 0,00 \\
\hline $\begin{array}{l}\text { Sexo** } \\
\text { (masculino) }\end{array}$ & $88,2 \%$ & $47,8 \%$ & 0,01 \\
\hline $\begin{array}{l}\text { Atraso escolar } * * * \\
\text { (anos) }\end{array}$ & 2,53 & 0,13 & 0,00 \\
\hline Anos de escolaridade & 6,2 & 6,8 & 0,34 \\
\hline $\begin{array}{l}\text { Anos de escolaridade do } \\
\text { cuidador }\end{array}$ & 7,7 & 8,5 & 0,62 \\
\hline $\begin{array}{l}\text { Renda mensal } \\
\text { (salários-mínimos) }\end{array}$ & 5,5 & 6,3 & 0,96 \\
\hline Número de pessoas na casa & 4,4 & 4,2 & 0,68 \\
\hline Número de filhos & 3,1 & 2,3 & 0,10 \\
\hline $\begin{array}{l}\text { Frequiência à escola } \\
\text { Sim } \\
\text { Não }\end{array}$ & $\begin{array}{l}82,4 \% \\
17,6 \%\end{array}$ & $100 \%$ & 0,07 \\
\hline $\begin{array}{l}\text { Escolaridade } \\
3^{\mathrm{a}}-4^{\mathrm{a}} \text { série } \\
5^{\mathrm{a}}-6^{\mathrm{a}} \text { série } \\
7^{\mathrm{a}}-8^{\mathrm{a}} \text { série } \\
1^{\mathrm{o}}-2^{\mathrm{o}} \text { ensino médio } \\
3^{\mathrm{o}} \text { ensino médio }\end{array}$ & $\begin{array}{c}23,5 \% \\
35,2 \% \\
29,4 \% \\
11,8 \% \\
\ldots \ldots\end{array}$ & $\begin{array}{c}4,3 \% \\
52,1 \% \\
26,1 \% \\
8,7 \% \\
8,7 \%\end{array}$ & 0,554 \\
\hline $\begin{array}{l}\text { Grupo étnico } \\
\text { Branco } \\
\text { Negro } \\
\text { Pardo }\end{array}$ & $\begin{array}{l}58,8 \% \\
23,5 \% \\
17,6 \%\end{array}$ & $\begin{array}{c}69,6 \% \\
17,4 \% \\
4,3 \%\end{array}$ & 0,883 \\
\hline $\begin{array}{l}\text { Tipo de família* } \\
\text { Monoparental } \\
\text { Biparental } \\
\text { Extensa } \\
\text { Abrigado }\end{array}$ & $\begin{array}{l}35,3 \% \\
29,4 \% \\
23,5 \% \\
11,8 \%\end{array}$ & $\begin{array}{c}21,7 \% \\
73,9 \% \\
4,3 \%\end{array}$ & 0,04 \\
\hline $\begin{array}{l}\text { Instrução do cuidador } \\
1^{\mathrm{a}}-4^{\mathrm{a}} \text { série } \\
5^{\mathrm{a}}-8^{\mathrm{a}} \text { série } \\
\text { Ensino médio incompleto } \\
\text { Ensino médio completo } \\
\text { Superior incompleto } \\
\text { Superior completo }\end{array}$ & $\begin{array}{c}35,3 \% \\
23,5 \% \\
11,8 \% \\
11,8 \% \\
\ldots \\
17,6 \%\end{array}$ & $\begin{array}{c}22,2 \% \\
16,7 \% \\
5,6 \% \\
38,9 \% \\
5,6 \% \\
11,1 \%\end{array}$ & 0,48 \\
\hline $\begin{array}{l}\text { Psicopatologia } * \\
\text { Frequiência } \\
\text { Sim } \\
\text { Não }\end{array}$ & $\begin{array}{l}35,3 \%(6) \\
52,9 \%(9)\end{array}$ & $\begin{array}{c}8,7 \%(2) \\
73,9 \%(17)\end{array}$ & 0,05 \\
\hline $\begin{array}{l}\text { Discórdia conjugal } \\
\text { Freqüência } \\
\text { Sim } \\
\text { Não }\end{array}$ & $\begin{array}{c}11,8 \%(2) \\
76,5 \%(13)\end{array}$ & $\begin{array}{l}13 \%(16) \\
69,6 \%(3)\end{array}$ & 0,62 \\
\hline
\end{tabular}

A Tabela 1 apresenta a comparação dos dados demográficos entre os grupos de adolescentes infratores e não-infratores. Foram encontradas diferenças significativas do ponto de vista estatístico 
entre os dois grupos em relação às variáveis idade, sexo, atraso escolar, tipo de família e presença de psicopatologia. Com relação ao sexo, houve uma proporção maior de meninos no grupo de adolescentes infratores (15 do sexo masculino e 2 do sexo feminino). Um estudo brasileiro encontrou uma relação de quatro meninos para apenas uma menina com comportamentos anti-sociais (Conte, 1996).

A idade, o tempo de atraso escolar e a porcentagem de famílias monoparentais foram significativamente maiores para o grupo de adolescentes infratores. Foi encontrada uma grande defasagem escolar entre os adolescentes infratores, sendo que, dos adolescentes que tinham idade para terem concluído o ensino fundamental, quinze não o fizeram. Estes dados estão de acordo com o estudo de Volpi (1997), o qual estudou 4.245 adolescentes infratores e observou que $96,6 \%$ da sua amostra não haviam concluído o Ensino Fundamental. Deste montante, $15,4 \%$ eram analfabetos.

Não foram encontradas diferenças significantes entre os dois grupos de adolescentes em termos das seguintes variáveis: frequiência à escola, renda mensal, grupo étnico, número de filhos, discórdia conjugal, tipo de moradia, anos de escolaridade do cuidador e instrução do cuidador.

\section{Tipos de delitos dos adolescentes infratores}

Como pode ser observado na Tabela 2 , dentre as infrações praticadas pelos adolescentes infratores, nove haviam se envolvido com porte de entorpecentes, quatro haviam praticado furto, três, assalto e um, lesão corporal dolosa.

A incidência maior de porte de entorpecentes, seguida pelo furto e assalto, assemelha-se aos dados encontrados por Silva e Rosseti-Ferreira (2002).

Tabela 2. Freqüência de Delitos do Grupo de Adolescentes Infratores.

\begin{tabular}{l|c|c}
\hline Tipo de Delito & N & \% \\
\hline Porte de entorpecente & 9 & 52,9 \\
Furto & 4 & 23,5 \\
Assalto & 3 & 17,6 \\
\hline
\end{tabular}

\section{Índice de Adversidade Familiar}

A diferença entre os índices médios de adversidade familiar de cada um dos grupos de adolescentes foi significativa $(p=0,025)$, indicada pelo teste U de Mann-Whitney. Para o grupo de adolescentes infratores, o índice médio de adversidade familiar foi 0,28 , e para o grupo de adolescentes nãoinfratores, 0,09.
Não obstante, apenas um dos cinco itens avaliados no Índice de Adversidade Familiar está presente significativamente em maior grau no ambiente familiar dos adolescentes infratores: a presença de psicopatologia na família (vide Tabela 1). Os demais itens do índice - baixa renda, número excessivo de filhos, número excessivo de pessoas morando na mesma casa e discórdia conjugal apresentam pontuação que individualmente não mostra diferença significante do ponto de vista estatístico entre os dois grupos de adolescentes.

Discordância entre a autopercepção dos adolescentes e de seus pais sobre problemas de comportamento destes.

A fim de verificar o grau de concordância entre a percepção dos pais e a autopercepção dos adolescentes acerca de problemas de comportamento do adolescente, foi calculado o índice de correlação de Pearson para as respostas apresentadas pelos pais e pelos adolescentes nos 89 itens de problemas de comportamento similares nos instrumentos preenchidos por eles (CBCL e YSR, respectivamente). Utilizou-se a sigla RI para referir-se a esse índice de correlação interitens do CBCL e YSR.

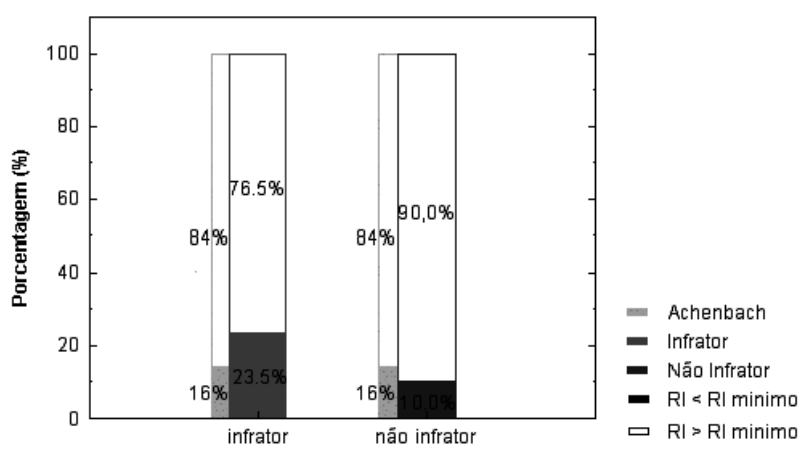

RI: Correlação inter-itens do CBCL e YSR.

RI mínimo: RI Situado a um desvio-padrão abaixo da média de RI obtida por Achenbach.

Figura 1. Porcentagem de pais e adolescentes discordantes no CBCL e no YSR para os grupos de adolescentes infratores e não-infratores em comparação com os pais e adolescentes da amostra normativa de Achenbach.

A fim de se ter um parâmetro acerca do grau de concordância nas correlações obtidas, os RIs foram comparados aos do estudo normativo feito por Achenbach (1991a). Os pais e adolescentes que se situavam em um desvio-padrão abaixo da média de RI obtida por Achenbach foram considerados "discordantes". A Figura 1 apresenta a porcentagem 
de pais e adolescentes discordantes para cada um dos grupos de adolescentes.

Nos estudos de Achenbach (1991a), a porcentagem dos escores de discordância nas somas das escalas de problemas de comportamento, segundo a percepção dos pais e dos próprios adolescentes, foi de $16 \%$. No grupo de adolescentes infratores brasileiros, a porcentagem de discordância foi de
23,5\% e, no grupo de adolescentes não-infratores, o índice foi de $10 \%$, com desvio-padrão abaixo da média. Apesar de a porcentagem de discordância no grupo de adolescentes infratores ter sido quase duas vezes maior do que a do grupo de adolescentes nãoinfratores, essa diferença não foi estatisticamente significativa $(p=0,296)$, como indicado pelo teste de Fisher.

Tabela 3. Escores Médios de DI, DE e DT no CBCL e no YSR Para os Grupos de ADOLESCENTES INFRATORES e Não-Infratores, $e$ Valor-p do teste T de Student.

\begin{tabular}{|c|c|c|c|c|c|c|c|c|c|}
\hline & \multicolumn{3}{|c|}{ Distúrbios Internalizantes (DI) } & \multicolumn{3}{|c|}{ Distúrbios Externalizantes (DE) } & \multicolumn{3}{|c|}{ Distúrbios Totais (DT) } \\
\hline & Infrator & Não- infrator & $p$ & Infrator & Não- infrator & $p$ & Infrator & Não- infrator & $p$ \\
\hline CBCL & 65,1 & 64,4 & 0,853 & 62,6 & 56,4 & 0,089 & 64,7 & 63,7 & 0,736 \\
\hline YSR & 59,2 & 53,8 & 0,082 & 52,3 & 50,7 & 0,640 & 56,5 & 52,8 & 0,225 \\
\hline $\mathbf{P}$ & 0,116 & $0,001 * * *$ & & $0,010 * *$ & 0,074 & & 0,028 & $0,000 * * *$ & \\
\hline
\end{tabular}

$* * \mathrm{p}<0,01$

$* * * \mathrm{p}<0,001$

Na Tabela 3 podem-se observar os escores médios de DI, DE e DT para os dois grupos de adolescentes obtidos tanto no questionário respondido pelos pais quanto no de autopercepção dos adolescentes. O exame da tabela permite analisar melhor o grau de discordância quanto aos escores médios obtidos em cada um dos dois grupos. Os escores médios de DT mostram que os pais reportaram mais problemas de comportamento do que os próprios adolescentes. Os escores médios de DT no CBCL alcançaram a categoria clínica (> 63), enquanto os escores médios de DT no YSR enquadraram-se na categoria normal. Essa diferença nos escores de DT no CBCL e YSR, porém, foi estatisticamente significativa apenas para a amostra de adolescentes não-infratores. Não foram encontradas diferenças estatisticamente significativas entre os escores médios de DT no CBCL entre os dois grupos, nem entre os escores médios de DT no YSR, ou seja, em termos de média do escore de distúrbios totais, os dois grupos não apresentaram diferenças. Essa similaridade apresenta-se nos escores médios de DI e de DE tanto no CBCL quanto no YSR.

As diferenças entre os grupos aparecem quando se comparam os escores de DI e de DE em termos de concordância da percepção dos pais e dos adolescentes. Na amostra de adolescentes nãoinfratores, o escore médio de DI foi significativamente maior na percepção dos pais do que na percepção dos adolescentes. Essa diferença não foi estatisticamente significativa no grupo de adolescentes infratores. Em síntese, os pais de adolescentes não-infratores reportaram mais problemas internalizantes do que seus filhos, enquanto os pais de adolescentes infratores reportaram tantos problemas internalizantes quanto seus filhos. Já quanto ao escore médio de DE, a diferença entre a percepção dos pais e a dos adolescentes foi significativa apenas para a amostra de adolescentes infratores, com os pais reportando uma maior taxa de problemas externalizantes.

\section{DISCUSSÃO}

O Índice de adversidade familiar no grupo de adolescentes infratores foi significativamente maior do que o índice do grupo de adolescentes não-infratores. Esses resultados estão de acordo com os estudos de Patterson et al. (1992) e Silvares (1993), fato de muito valor, pois demonstra o poder heurístico do índice quantificado pela primeira vez em nossa cultura, além de confirmar trabalhos anteriores brasileiros e estrangeiros.

$\mathrm{O}$ ambiente familiar da amostra dos adolescentes infratores apresentou um maior número de fatores considerados adversos ao desenvolvimento. É interessante observar, contudo, que essa diferença não foi devida à renda ou às condições de moradia, mas sim, a fatores que em conjunto com outras variáveis podem interferir ainda mais diretamente na qualidade das relações afetivas, como, por exemplo, a presença de psicopatologia na família (Rodrigo \& Palácios, 1998; Rutter,1981; Patterson et al., 1992).

Considerando os fatores que compõem o Índice de Adversidade Familiar, além da presença de psicopatologia, não foi encontrada diferença significativa entre os grupos de adolescentes infratores e não-infratores. Chama a atenção especialmente a falta de diferença entre os grupos quanto à freqüência 
de discórdia conjugal. Acredita-se que o fato de 76,5\% dos pais do grupo de adolescentes infratores não apontarem discórdia conjugal pode ser explicado pela predominância das famílias monoparentais neste grupo. Nas famílias de adolescentes infratores compostas por pai/padrasto e mãe havia relatos de brigas com agressão física, associadas quase sempre ao uso de álcool e drogas.

Ressaltamos que o ambiente adverso será potencialmente prejudicial à saúde do adolescente se ele for uma pessoa vulnerável. Alguns adolescentes inseridos em ambientes adversos conseguem superar essas dificuldades; em contrapartida, outros adolescentes inseridos em famílias sem a presença de fatores adversos, mas extremamente vulneráveis, podem apresentar problemas comportamentais (Silva \& Rosetti-Ferreira, 2002).

Quanto ao grau de discordância da autopercepção e da percepção dos pais sobre problemas de comportamento, não foi encontrada diferença significativa entre os dois grupos de adolescentes de acordo com o teste estatístico aplicado. No entanto, é importante frisar que, em termos percentuais, o grau de discordância entre o grupo de pais e adolescentes infratores foi de $23,5 \%$ - mais que o dobro do observado no grupo de pais e adolescentes nãoinfratores (10\%). É possível que a falta de significância estatística deva-se ao número reduzido de participantes.

Tanto no grupo de adolescentes infratores quanto no de adolescentes não-infratores os pais reportaram mais problemas de comportamento. No caso da amostra de adolescentes infratores, esses resultados estão de acordo com os de Butler et al. (1995) e Glaser et al. (2000). Parece relevante discuti-los, tendo-se em vista os resultados do estudo de Conte (1996), no qual o YSR foi respondido por adolescentes infratores em dois momentos: no contato inicial e após um período de intervenção. Os adolescentes indicaram mais problemas de comportamento no segundo momento, resultado que a autora relaciona à confiança estabelecida durante o processo de intervenção. No presente estudo, por conta da estrutura institucional e da dificuldade de os adolescentes comparecerem à perícia psicossocial, o YSR foi aplicado no primeiro contato com estes.

Apesar de os escores no CBCL terem sido mais elevados do que os escores do YSR em ambos os grupos, a diferença nos escores médios de Distúrbios totais no CBCL e YSR foi estatisticamente significativa apenas para a amostra de adolescentes não-infratores. Tanto adolescentes que estão respondendo pela prática de atos infracionais, aguardando decisão judicial, quanto seus pais, temem a medida de privação de liberdade. Nossa experiência pessoal mostra que os pais tentam minimizar os comportamentos anti-sociais de seus filhos e a gravidade das infrações por eles praticadas. Já os pais dos adolescentes não-infratores estavam em busca de atendimento médico, nutricional, psicológico, portanto estavam mais livres para responder ao instrumento. A interpretação desses resultados fica prejudicada pelo fato de a literatura nacional a respeito do tema ser carente e haver poucos estudos sobre os dois instrumentos de avaliação utilizados (ambos ainda sem normas brasileiras).

Acreditamos que esses resultados indicam a importância da implementação de programas de prevenção de comportamentos de risco para a população infanto-juvenil, tais como o de Trianes (1994) e Roche (2004). Estes programas visam ao fortalecimento dos fatores protetores ao ciclo vital, como a estimulação de sentimentos empáticos, o desenvolvimento de habilidades sociais e a autoestima.

Por fim, cabe apontar algumas limitações metodológicas do estudo, tais como o número reduzido de participantes e o fato de a amostra de adolescentes não-infratores ser proveniente de um ambulatório médico. Apesar disso, consideramos que o presente trabalho cumpriu com os objetivos a que se propôs e abriu espaço para novas pesquisas que possam aprofundar os resultados encontrados.

\section{REFERÊNCIAS}

Achenbach, T. M. (1991a). Integrative guide for de 1991 CBCL 418, YSR and TRF profiles. Burlington: University of Vermont.

Achenbach, T. M. (1991b). Manual for the youth self-report and 1991 profile. Burlington: University of Vermont.

Alvarenga, P., Pacheco, J., Reppold, C., Piccinini, C. A., \& Hutz, C. S. (2005). Estabilidade do Comportamento Anti-social na Transição da Infância Para a Adolescência: uma perspectiva desenvolvimentista. Psicologia, Reflexão e Crítica, 18(1), 5561.

Azevedo, M. A., \& Guerra, V. (Orgs.). (1989). Crianças vitimizadas - A síndrome do pequeno poder. São Paulo: Iglu.

Bordin, I. S., Mari, J. J., \& Caeiro, M. F. (1995). Validação da versão brasileira do Child Behavior Checklist - Inventário de Comportamentos da Infância e Adolescência: dados preliminares. Revista Associação Brasileira de Psiquiatria, 17(2), 55-66.

Butler, S. M., Mackay, S. A., \& Dickens, S. E. (1995). Maternal and adolescent ratings of psychopathology in young offender and non-clinical males. Canadian Journal of Behavioural Science, 27(3), 333-342. 
Conte, F. C. S. (1996). Pesquisa e intervenção clínica em comportamentos delinqüentes numa comunidade pobre. Tese de Doutorado não publicada, Instituto de Psicologia, Universidade de São Paulo, São Paulo.

Glaser, B. A., Calhoun, G. B., Bradshaw, C. P., Bates, J. M., \& Socherman, R. E. (2001). Multi-observer assessment of problem behavior in adjudicated youths: Patterns of discrepancies. Child \& Family Behavior Therapy, 23(2), 33-45.

Patterson, G. R., Reid, J. B., \& Dishion, T. J. (1992). A social interactional approach to antisocial boys (Vol. 4). United States of America: Copyright.

Roche-Olivar, R. (2004). Inteligencia Prosocial: Educación de las emociones y valores. Universitat Autónoma de Barcelona Bellaterra: Servei de Publicacions.

Rodrigo, M. J., \& Palacios, J. (Orgs.). (1998). Família y desarrollo humano: Psicología y Educación. Madrid: Alianza Editorial.

Rutter, M. (1981). Stress, coping and development: Some issues and questions. Journal of Child Psychiatry, 22, 323-356.

SEADE (1999). Pesquisa de condições de vida. São Paulo: Fundação Sistema Estadual de Análise de Dados.
Silvares, E. F. M. (1993). O papel preventivo das clínicas-escola de Psicologia no seu atendimento a crianças. Temas de Psicologia, 2, 87-97.

Silvares, E. F. M. (2000). Terapia comportamental com famílias de crianças agressivas: porque, como e quando. Paidéia: Cadernos de Psicologia e Educação, 20, 24-32.

Silva, P., \& Rossetti-Ferreira, C. (2002). Continuidade/ descontinuidade no envolvimento com o crime: uma discussão crítica da Literatura na Psicologia do Desenvolvimento. Psicologia Reflexão e Crítica, 18, 55-61.

Trianes, M. V. (1994). Educación de competencia para las relaciones interpersonales en niños de compensatória Derechos de los niños y escuela. Infancia y Sociedad, 27/28.

Volpi, M. (1997). O adolescente e o ato infracional. São Paulo: Cortez.

Recebido em 24/10/2006 Aceito em 15/04/2007

Endereço para correspondência : Maria Delfina Farias Dias Tavares da Silva. Av. Manoel da Nóbrega, 451, AP. 2C, Itararé, CEP 11320-200. São Vicente-SP. E-mail: ludel@terra.com.br 